\title{
Effectiveness of the mindfulness-acceptance-commitment-based approach on athletic performance and sports competition anxiety: a randomized clinical trial
}

Mahmood Dehghani ${ }^{1}$, Azadeh Delbar $\mathrm{Saf}^{2}$, Asghar Vosoughi ${ }^{3}$, Giti Tebbenouri ${ }^{3}$, Hadi Ghazanfari Zarnagh ${ }^{4}$

${ }^{1}$ Ph.D. of Clinical Psychology, Assistant Professor, Tehran Institute of Psychiatry- School of Behavioral Sciences and Mental Health, Iran University of Medical Sciences, Tehran, Iran

${ }^{2}$ M.Sc. of Clinical Psychology, Islamic Azad University of Ahvaz, Ahvaz, Iran

${ }^{3}$ M.Sc. of Clinical Psychology, University of Tabriz, Tabriz, Iran

${ }^{4} \mathrm{Ph} . \mathrm{D}$. Candidate in Clinical Psychology, Tehran Institute of Psychiatry- School of Behavioral Sciences and Mental Health, Iran University of Medical Sciences, Tehran, Iran

Type of article: Original

\begin{abstract}
Background: Improving sports performance and reducing anxiety is one of the most important goals of athletes. Recurrence of symptoms and treatment cessation are common problems with common interventions. Approaches based on mindfulness-acceptance-commitment (MAC) can be a response to these limitations

Objective: The main purpose of the present study was to determine effectiveness of the mindfulness-acceptancecommitment-based approach to athletic performance enhancement and sports competition anxiety in students who have had athletic experience for 3 to 5 years.

Methods: This randomized clinical trial was conducted at the Faculty of Educational Sciences of Iran University of Medical Sciences in Tehran, Iran, from May 1, 2017 to September 15, 2017. A total of 31 students were randomly assigned to experimental $(\mathrm{n}=15)$ and control groups $(\mathrm{n}=16)$. The experimental group received the protocol Mindfulness-Acceptance-Commitment (MAC) for 8 sessions. Subjects completed the Charbonneau Sports Performance Questionnaire, Action and Acceptance Questionnaire (AAQ) and Sports Competition Anxiety Test (SCAT) Questionnaire. Data analysis was conducted by using multivariate covariance analysis (MANCOVA) by SPSS-22.

Results: The results of the study indicated that the MAC approach increases significantly the performance of basketball playing athletes $(\mathrm{p}<0.05)$. Furthermore, the MAC approach decreases significantly experiential avoidance and sports anxiety in athletes $(\mathrm{p}<0.05)$. The size of the difference between the groups is moderate (Eta squared).

Conclusions: This study revealed that the mindfulness-acceptance-commitment-based approach is an effective intervention to increasing athletic performance and reducing experiential avoidance and sports anxiety in athletes.

Trial registration: The trial was registered at the Iranian Registry of Clinical Trials (http://www.irct.ir) with the Irct ID: IRCT20170409033333N1.

Funding: This study has been financially supported by the Research Deputy of Iran University of Medical Sciences (Code: 95-04-185-29333).

Keywords: Mindfulness-Acceptance-Commitment-Based approach, Athletic performance, Sports anxiety
\end{abstract}

\section{Introduction}

Athletes take great efforts to improve their athletic performance and compensate the barriers to their athletic performance (1). Studies show that academic athletes suffer from psychological problems (2). One of the most important stress factors in student athletes is time-related factors (3). Student athletes do not have enough time to attend academic and sports activities at the same time. Also, problems in interpersonal relations, depression, stress,

\section{Corresponding author:}

Hadi Ghazanfari Zarnagh, Tel: +98.9126382197, Fax: +98. 66506853, Email: Ghazanfari.hadi67@gmail.com

Received: November 04, 2017, Accepted: December 27, 2017, Published: May 2018

iThenticate screening: December 20, 2017, English editing: April 07, 2018, Quality control: April 15, 2018

This article has been reviewed / commented by three experts

(C) 2018 The Authors. This is an open access article under the terms of the Creative Commons Attribution-NonCommercialNoDerivs License, which permits use and distribution in any medium, provided the original work is properly cited, the use is noncommercial and no modifications or adaptations are made. 
and competitive anxiety are higher in athletes (1). Several psychological interventions such as cognitive-behavioral therapy, mental imagery, relaxation, and other psychological interventions have been used to increase athletic performance, and reduce stress, competitive anxiety and other problems of athletes, and their effectiveness has been confirmed (4). But these treatments have limitations, the most common of which include recurrence of symptoms and treatment cessation (5). Also, previous treatments usually try to change the cognitive and emotional content of athletes. These treatments try to change the negative thoughts and emotions of the students. Psychological changes in individuals cause resistance from a psychological point of view in the short term and lead to treatment cessation (6). These treatments also emphasize less on interpersonal relationships and quality of life (7). So, evaluating effectiveness of new interventions is essential. Mindfulness and acceptance-based intervention is one of the new areas recently introduced in sport psychology to improve athletic performance (1). Mindfulness in the definition given by KabatZinn consists of two processes of flexible attention and non-judgment awareness (7). Mindfulness means the acceptance and awareness of thoughts, emotions, physical senses, and external experiences at the present time without judging and trying to control and suppress them. Such a view helps a person to accept his negative thoughts and unpleasant emotions without controlling or avoiding them. This acceptance helps the person to proper function in stressful situations, and in countering negative thoughts and unpleasant emotions $(1,7)$. Mindfulness and acceptancebased interventions help to change the type of person's communication with negative thoughts and emotions in different situations (7). Using mindfulness exercises, acceptance, identifying values and changing value-based behavior facilitates the ability to accept negative thoughts and emotions, and increases the cognitive flexibility and metacognitive control of the individual and, thus improves the person's sports performance (8). The mechanisms of change in mindfulness-based interventions have been studied. Exposure, cognitive change, self-management, relaxation and acceptance have been identified as mechanisms of change (9). Mindfulness and acceptance-based interventions can be used to increase concentration, improve working memory, reduce stress and negative thoughts, increase athletic performance and the athlete's speed (10-13). Studies show that mindfulness increases athletes' speed by reducing the person's avoidance of negative thoughts and unpleasant emotions (8). Mindfulness strategies reduce the anxiety and perfectionism of athletes $(9,10)$, because mindfulness decreases the suppression of negative thoughts, which reduces the person's avoidance and reduces the cycle of the influx of negative thoughts during sports events. Studies have shown that mindfulness can increase an athlete's commitment to exercise (11). In mindfulness and acceptance-based intervention, by specifying the values and goals of athletes in life and in sport, their commitment and motivation to continue sport and progress are increased (11). Despite the many studies on the theoretical basis of the effect of mindfulness-based treatments on exercise performance of athletes, less research has examined the effectiveness of mindfulness and acceptance-based intervention in a controlled and interventional manner. Therefore, research in this field seems necessary. The main purpose of the present study was to determine the effectiveness of the mindfulness-acceptance-commitment-based approach to athletic performance enhancement and sports competition anxiety in student athletes.

\section{Material and Methods}

\subsection{Trial design}

The present study was a randomized clinical trial that was conducted from May 1, 2017 to September 15, 2017.

\subsection{Participants}

This study was conducted at the Faculty of Educational Sciences at Iran University of Medical Sciences in 2017. The participants in the study were female athletes and were studying at the Iran University of Medical Sciences and Tehran University of Medical Sciences, and had two training sessions a week and were preparing for sports competitions. Athletes were from different fields of study and medical education, and their age range was between 18 and 30. Frist, psychiatric interviews based on DSM-5 were conducted for research sample, and subjects suffering from mental disorders were excluded from the study. After coordination with the Faculty of Education, intervention sessions began.

\subsection{Interventions}

In this study, after the randomization of the subjects, the experimental group was intervened based on the Moore and Gardner MAC Protocol, summarized in Table 1, conducted for 8 sessions of 1.5 hours and grouped in the physical education faculty of Iran University of Medical Sciences by a Ph.D. student of clinical psychology who had passed the MAC curriculum (11). The control group did not receive any intervention. Subjects completed the research questionnaires before and after the intervention. 
Table 1. The content of the intervention sessions

\begin{tabular}{|c|c|}
\hline Session & Setting agenda \\
\hline \multirow[t]{6}{*}{1} & $\begin{array}{l}\text { Implementation of pre- test included Assessment of, Athletic Performance, the } \\
\text { Sport Competition Anxiety and Experiential avoidance }\end{array}$ \\
\hline & Introducing Mindfulness-Acceptance-Commitment-Based Approach \\
\hline & Assessment of One's Relationship with Internal Experiences \\
\hline & $\begin{array}{l}\text { Challenging the control of negative thoughts and emotions (control as the } \\
\text { problem: creative hopelessness ) and the Destructive Effects of Experiential } \\
\text { Avoidance }\end{array}$ \\
\hline & Introductory Breathing Exercise \\
\hline & Introduction to self-monitoring \\
\hline \multirow[t]{3}{*}{ 2-3 (Mindfulness phase) } & Continuing Previous exercises \\
\hline & Introducing Mindfulness and Cognitive defuse \\
\hline & $\begin{array}{l}\text { To teach mindfulness exercises included: Body scan practice, The Three Minute } \\
\text { Breathing Space and Mindfulness practice in everyday life }\end{array}$ \\
\hline \multirow{4}{*}{$\begin{array}{l}4-5 \text { (Values identification and } \\
\text { Commitment Phase) }\end{array}$} & Continuing Previous exercises \\
\hline & Introducing Values and Values-Driven Behavior \\
\hline & Assessment of Values, Goals, Actions, and Barriers \\
\hline & Increasing Engagement in Valued Activities \\
\hline \multirow[t]{2}{*}{ 6-7 (Acceptance Phase) } & Introducing Acceptance and Enhancing Commitment: \\
\hline & Cultivating Acceptance through Mindfulness skills and metaphors \\
\hline \multirow{2}{*}{$\begin{array}{l}8 \text { (Integration and Practice } \\
\text { Phase) }\end{array}$} & Maintaining and Enhancing Mindfulness, Acceptance, and Commitment \\
\hline & $\begin{array}{l}\text { Implementation of post- test included Assessment of, Athletic Performance, the } \\
\text { Sport Competition Anxiety and Experiential avoidance }\end{array}$ \\
\hline
\end{tabular}

\subsection{Selection Criteria}

The present study inclusion criteria were being a member of the university sports team, age range between 18 and 30 years and absence of acute and debilitating mental disease. Exclusion criteria were having a psychiatric disorder and not being a student athlete.

\subsection{Sample Size}

The statistical population of the study was athletic students of Iran University of Medical Sciences and Tehran University of Medical Sciences. The women's basketball team of both Iran and Tehran University of Medical Sciences were selected as 37 people for the research sample. The sample size of the present study was calculated based on previous studies $(1,4)$ and by considering the test power of $80 \%$ and a confidence level of $95 \%$. In the present study, psychiatric disorder was one of the most important confounding variables. Subjects with a specific psychiatric disorder were excluded from the study through psychiatric interviews by clinical psychologist. Finally, the study samples $(\mathrm{n}=$ $31)$ were randomly divided into two experimental $(n=15)$ and control groups $(n=16)$ by random numbers generation.

\subsection{Research ethics}

The research was approved by the Ethics Committee of Iran University of Medical Sciences (Code: IR.IUMS.REC 1395.95-04-185-29333). In order to observe the ethics of research, the MAC approach was taught to control group subjects after the intervention. Also, Subjects completed the form of informed consent before the intervention. The subjects were informed about the objective of the study and that participation in the research is voluntary for subjects. Also, the trial was registered at the Iranian Registry of Clinical Trials (http://www.irct.ir) with the Irct ID: IRCT20170409033333N1.

\subsection{Outcomes and tools}

The research variables included Sports Performance, Experiential Avoidance and Sports Competition Anxiety. The research tools included:

2.7.1. Charbonneau Sports Performance Questionnaire (SPQ):

The questionnaire was made in 2001 by Charbonneau (13). The questionnaire was developed using a five-point Likert scale and was designed to evaluate the performance of athletes. Scores range was from 5 to 25 . High scores indicated high performance. Low scores indicated low performance. In this study, we translated the Charbonneau Sports Performance Questionnaire (SPQ) to Persian language and found appropriate formal validity by six experts in sport 
psychology. To determine the reliability of the questionnaire, we tested it on 50 dormitory students of Iran University of Medical Sciences. In this sample, the SPQ demonstrated appropriate reliability $(\alpha=0.91)$. The questionnaire was used to assess exercise performance.

2.7.2. Action and Acceptance questionnaire (AAQ):

The questionnaire of Action and Acceptance assesses the degree of avoidance of internal experiences. The questionnaire has 9 items. High scores in subjects, show avoidance of internal experiences and low scores, show acceptance of internal experiences. The score for this questionnaire is between 9 and 63 . Internal consistency and reliability of retest of the questionnaire are reported as appropriate in the Iranian samples (14). In this study, this questionnaire was used to determine the extent of avoiding internal experiences.

2.7.3. Sport Competition Anxiety Test (SCAT) Questionnaire:

Martens Sport Competition Anxiety Test Questionnaire has 15 questions, and scores range from 10 to 30 . Higher scores represent higher anxiety. Martens obtained the main reliability coefficient of this test as 0.77 (15). In this study, we translated the Sports Competition Anxiety Test (SCAT) questionnaire to Persian language and found appropriate formal validity by six experts in sport psychology. To determine the reliability of the questionnaire, we tested this Questionnaire on 50 dormitory students of Iran University of Medical Sciences. In this sample, the SCAT demonstrated appropriate reliability $(\alpha=0.82)$. In this study, this questionnaire was used to assess athletic anxiety.

\subsection{Randomization and blinding}

In this clinical trial, after coordinating with the faculties of education in universities of Iran and Tehran, basketball teams were selected as a research sample $(n=37)$. By conducting a psychiatric interview, subjects with mental disorders were excluded from the study $(n=6)$. Randomization was done in two groups by random numbers generation in SPSS 22 software. Blinding has not been done, because it is impossible. Researcher and subjects are aware of the treatment process under any condition (5). The stages of study is illustrated in Figure 1.

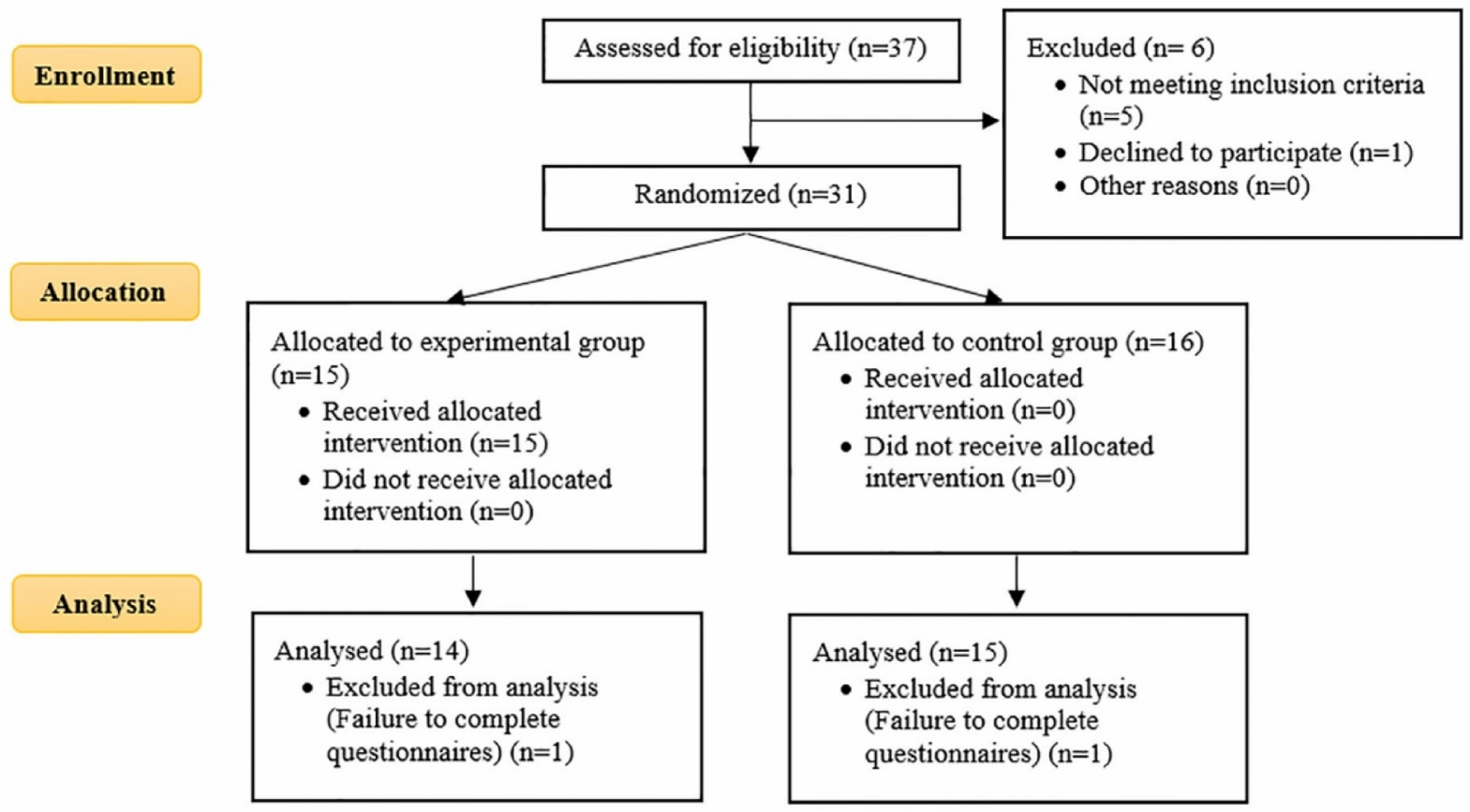

Figure 1. CONSORT diagram of the study

\subsection{Statistical Methods}

IBMC SPSS C Statistics version 22 (IBMC Corp., Armonk, NY, USA) was used to analyze the statistical data. The statistical methods for data analysis were descriptive statistics and multivariate covariance analysis (MANCOVA).

\section{Results}

The mean and standard deviation of the subjects' age in the experimental group was $23.44 \pm 0.49$ years old and in the control group it was $22.34 \pm 0.34$ years old. All subjects were female students. Subjects were from different disciplines of basic sciences of medicine and general medicine who were studying at different levels. The mean and standard 
deviation of athletic performance scores, experiential avoidance and anxiety in the pretest and posttest phases are shown in Table 2. Multivariate analysis of covariance analysis (MANCOVA) was used for data analysis. The Box's $\mathrm{M}$ and Levene's test was used to evaluate the assumptions of MANCOCA. Considering the significance level of 0.05 , the results of these two tests were not significant $(\mathrm{BOX}=31.765, \mathrm{~F}=1.69, \mathrm{p}=0.287)$. Therefore, MANCOVA can be used to analyze the data. Also, the results of the Wilk's Lambda test indicated that the effect of the group on exercise performance, experiential avoidance and anxiety is significant $(\mathrm{F}=18.765, \mathrm{p}=0.0045)$. Therefore, covariance test can be used. The results of the Wilk's Lambda test indicated that there is a significant difference between at least one of the variables of exercise performance, experiential avoidance and anxiety, between the two groups (Table 3). The results of MANCOVA in Table 4 indicated that by controlling the scores of pre-test, the MAC approach has a significant effect on variables of athletic performance, experience avoidance and sport anxiety, and there is a significant difference between the two groups and the MAC approach has significant effect on athletic performance and experiential avoidance and sport anxiety $(\mathrm{F}=0.672, \mathrm{~F}=0.473, \mathrm{~F}=0.724, \mathrm{p}=0.0032)$. The size of the difference between the groups is moderate (Effect of size: moderate).

Table 2. Mean and standard deviation of variables

\begin{tabular}{|l|l|l|l|l|l|l|l|l|}
\hline \multirow{3}{*}{ The dependent variable } & \multicolumn{3}{|l|}{ Experimental group } & \multicolumn{3}{l|}{ Control group } \\
\cline { 2 - 10 } & Pre-test & \multicolumn{2}{l|}{ Post-test } & \multicolumn{2}{l|}{ Pre-test } & \multicolumn{2}{l|}{ Post-test } \\
\cline { 2 - 10 } & M & SD & M & SD & M & SD & M & SD \\
\hline Sport performance & 12.5 & 4.52 & 19.66 & 3.12 & 13.44 & 4.65 & 14.65 & 3.89 \\
\hline Experiential avoidance & 36.44 & 8.55 & 28.33 & 6.45 & 26.33 & 6.77 & 28.86 & 7.34 \\
\hline Sport anxiety & 18.43 & 4.77 & 12.50 & 3.75 & 19.55 & 4.55 & 17.56 & 3.77 \\
\hline
\end{tabular}

Table 3. The results of Wilk's Lambda test

\begin{tabular}{|l|l|l|l|l|l|c|}
\hline Test & Value & $F$ & df error & df effect & Sig. & $\eta \overline{2}$ \\
\hline Wilk's Lambda & 0.384 & 18.765 & 29 & 2 & 0.001 & 0.345 \\
\hline \multicolumn{7}{|c|}{ A significant level is considered $\mathrm{p}<0.05$} \\
\hline
\end{tabular}

Table 4. The results of MANCOVA

\begin{tabular}{|l|l|l|l|l|c|}
\hline Variable & MS & df & F & Sig. & $n \overline{2}$ \\
\hline Sport performance & 54.45 & 1 & 0.672 & 0.001 & 0.586 \\
\hline Experiential avoidance & 43.44 & 1 & 0.473 & 0.001 & 0.423 \\
\hline Sport anxiety & 29.87 & 1 & 0.724 & 0.001 & 0.623 \\
\hline
\end{tabular}

MS: Mean square; A significant level is considered $\mathrm{p}<0.05$

\section{Discussion}

The main objective of the present study was to determine the effectiveness of approaches based on mindfulnessacceptance-commitment (MAC) on athletic performance and competitive anxiety in student athletes. The results of this study showed that the MAC approach improves athletic performance and reduces experiential avoidance and athletic anxiety in student athletes. The results of this study showed that acceptance of negative thoughts and emotions decreased in athletes, which is consistent with the findings of previous research $(1,11,13)$. As stated in this study, interventions based on acceptance and mindfulness increase awareness of internal, external and motor experiences during exercise. Mindfulness training helped female athletes to get out of their auto-guidance and mind-wandering. According to the therapies based on acceptance and mindfulness, as well as previous studies, in auto-guidance and mind-wandering, the individuals are not aware of their emotions and respond automatically and impulsively which results in a judicious attitude to these emotions and thoughts. The results of this study showed that the first stage of mindfulness is knowledge of these automated judgments, as the background of prejudices and negative thoughts. Mindfulness helped female athlete students to see negative thoughts as mere emotions and thought, and accept them. Acceptance also increased their athletic performance. The results of this study showed that experiential avoidance and competitive anxiety decreased in students. According to previous studies and MAC-based theories, experiential avoidance refers to the individual's lack of eagerness to experience internal events such as negative thoughts and emotions (16). There has been effort to avoid negative thoughts and emotions, but controlling and suppressing them will reveal negative thoughts and emotions, indeed, if we try to think more about what we do not want to think. Accepting is an alternative to experiential avoidance. Mindfulness skills increase the ability of acceptance. In acceptance, we accept negative thoughts and emotions without trying to be rid of them, and thus experience less negative emotions. In acceptance, we want to experience negative thoughts and emotions as they happen. So, 
acceptance does not mean enduring $(7,8)$. Previous studies have shown that the MAC approach is an appropriate intervention for athletes. But these studies have some limitations, most important of which include methodologic problems, small sample size, lack of controlling assignments between sessions, and lack of controlling psychological and personality problems $(17,18)$. Also, previous research did not use a single protocol, while one of the most important features of our study is the effectiveness of having a unique well-defined protocol (11). In the present study, female athletes with psychological and personality problems were excluded from the study through psychiatric interviews. Also, assignments between sessions were followed up by phone call, which increased the effectiveness of intervention and reduced the rate of leaving the research. In the present study, leaving the research was less prevalent than previous studies. Usually, in experimental studies, the loss of participants is compensated by a high sample size or substitution of subjects. In explaining this finding, according to the theory of acceptance and mindfulness, one of the important components of the MAC approach is emphasis on behavioral change based on life values $(7,11)$. Given that exercise is one of the health-related values, athlete students committed to their sporting programs despite time conflicts between academic and sports activities. This issue can be helpful, especially for athletic students who are practicing professionally. This study had a few limitations, including limitations of the research samples to female athletes, lack of comparison with common interventions, and lack of comparison with other sports fields.

\section{Conclusions}

The results of this study showed that the Mindfulness-Acceptance-Commitment-Based Approach is an effective intervention to increase athletic performance and reduce experiential avoidance and sports anxiety in athletes. So, the Mindfulness-Acceptance-Commitment-Based Approach is evidence-based intervention for athletes and can be used in sports environments. It is recommended to use unit protocols, appropriate sample size, sports fields and monitoring of homework on mindfulness, between sessions in future research.

\section{Trial registration:}

The trial was registered at the Iranian Registry of Clinical Trials (http://www.irct.ir) with the Irct ID: IRCT20170409033333N1.

\section{Funding:}

This study has been financially supported by the Research Deputy of Iran University of Medical Sciences (Code: 9504-185-29333).

\section{Acknowledgments:}

We would like to thank Iran University of Medical Sciences research deputy who supported the present study.

\section{Conflict of Interest:}

There is no conflict of interest to be declared.

\section{Authors' contributions:}

All authors contributed to this project and article equally. All authors read and approved the final manuscript.

\section{References:}

1) Gardner FL, Moore ZE. Mindfulness-based and acceptance-based interventions in sport and performance contexts. Curr Opin Psychol. 2017; 16: 180-4. doi: 10.1016/j.copsyc.2017.06.001. PMID: 28813347.

2) Giurgiu RL, Damian M. Stress \& coping in athletes and non-athletes students-comparative study. Procedia Soc Behav Sci. 2015; 180: 332-7. doi: 10.1016/j.sbspro.2015.02.125.

3) Schaal K, Tafflet M, Nassif H, Thibault V, Pichard C, Alcotte M, et al. Psychological balance in high level athletes: gender-based differences and sport-specific patterns. PloS one. 2011; 6(5): e19007. doi: 10.1371/journal.pone.0019007. PMID: 21573222.

4) Reese LM, Pittsinger R, Yang J. Effectiveness of psychological intervention following sport injury. J Sport Health Sci. 2012; 1(2): 71-9. doi: 10.1016/j.jshs.2012.06.003.

5) Schottenbauer MA, Glass CR, Arnkoff DB, Tendick V, Gray SH. Nonresponse and dropout rates in outcome studies on PTSD: Review and methodological considerations. Psychiatry. 2008; 71(2): 134-68. doi: 10.1521/psyc.2008.71.2.134. PMID: 18573035.

6) Leichsenring F, Rabung S. Long-term psychodynamic psychotherapy in complex mental disorders: update of a meta-analysis. Br J Psychiatry. 2011; 199(1): 15-22. doi: 10.1192/bjp.bp.110.082776. PMID: 21719877. 
7) Roemer L, Orsillo SM. Mindfulness-and acceptance-based behavioral therapies in practice. New York: Guilford Press; 2010.

8) Van der Velden AM, Kuyken W, Wattar U, Crane C, Pallesen KJ, Dahlgaard J, et al. A systematic review of mechanisms of change in mindfulness-based cognitive therapy in the treatment of recurrent major depressive disorder. Clin Psychol Rev. 2015; 37: 26-39. doi: 10.1016/j.cpr.2015.02.001. PMID: 25748559.

9) Friese M, Messner C, Schaffner Y. Mindfulness meditation counteracts self-control depletion. Conscious Cogn. 2012; 21(2): 1016-22. doi: 10.1016/j.concog.2012.01.008. PMID: 22309814.

10) Moore ZE. Theoretical and empirical developments of the Mindfulness-Acceptance-Commitment (MAC) approach to performance enhancement. J Clin Sport Psycho. 2009; 3(4): 291-302. doi: 10.1123/jcsp.3.4.291.

11) Bernier M, Thienot E, Codron R, Fournier JF. Mindfulness and acceptance approaches in sport performance. J Clin Sport Psycho. 2009; 3(4): 320-33. doi: 10.1123/jcsp.3.4.320.

12) Scott - Hamilton J, Schutte NS, Brown RF. Effects of a mindfulness intervention on sports - anxiety, pessimism, and flow in competitive cyclists. Appl Psychol Health Well Being. 2016; 8(1): 85-103. doi: 10.1111/aphw.12063. PMID: 26970111.

13) Charbonneau D, Barling J, Kelloway EK. Transformational leadership and sports performance: The mediating role of intrinsic motivation. J Appl Soc Psychol. 2001; 31(7): 1521-34. doi: 10.1111/j.15591816.2001.tb02686.x.

14) Zargar F, Farid AA, Atef-Vahid MK, Afshar H, Omidi A. Comparing the effectiveness of acceptance-based behavior therapy and applied relaxation on acceptance of internal experiences, engagement in valued actions and quality of life in generalized anxiety disorder. J Res Med Sci. 2013; 18(2): 118. PMID: 23914213, PMCID: PMC3724371.

15) Martens R, Vealey RS, Burton D. Competitive anxiety in sport. Champaign: Human kinetics; 1990.

16) Josefsson T, Ivarsson A, Lindwall M, Gustafsson H, Stenling A, Böröy J, et al. Mindfulness Mechanisms in Sports: Mediating Effects of Rumination and Emotion Regulation on Sport-Specific Coping. Mindfulness. 2017; 8(5): 1354-63. doi: 10.1007/s12671-017-0711-4. PMID: 28989551 , PMCID: PMC5605575.

17) Lynn SJ, Malakataris A, Condon L, Maxwell R, Cleere C. Post-traumatic stress disorder: cognitive hypnotherapy, mindfulness, and acceptance-based treatment approaches. Am J Clin Hypn. 2012; 54(4): 311 30. doi: 10.1080/00029157.2011.645913. PMID: 22655333.

18) Buhlmayer L, Birrer D, Rothlin P, Faude O, Donath L. Effects of Mindfulness Practice on PerformanceRelevant Parameters and Performance Outcomes in Sports: A Meta-Analytical Review. Sports Med. 2017; 47(11): 2309-21. doi: 10.1007/s40279-017-0752-9. PMID: 28664327. 